$\begin{array}{ll}\text { Research Square } & \text { Preprints are preliminary reports that have not undergone peer review. } \\ \text { They should not be considered conclusive, used to inform clinical practice, } \\ \text { or referenced by the media as validated information. }\end{array}$

\title{
The Natural History of the Black Soldier Fly, Hermetia Illucens: Insights From Complete Mitochondrial Genome Sequences
}

\author{
Joseph Guilliet ( $\square$ joseph.guilliet@gmail.com ) \\ Évolution, Génomes, Comportement, Écologie \\ Guillaume Baudouin \\ Cycle Farms \\ Pollet Nicolas \\ Évolution, Génomes, Comportement, Écologie \\ Filee Jonathan \\ Évolution, Génomes, Comportement, Écologie
}

\section{Research Article}

Keywords: Hermetia illucens, phylogeny, mitochondrial genome, phylogeography

Posted Date: February 11th, 2022

DOI: https://doi.org/10.21203/rs.3.rs-1250528/v1

License: () (1) This work is licensed under a Creative Commons Attribution 4.0 International License. Read Full License 


\section{Abstract}

\section{Background}

The Black Soldier Fly (BSF) Hermetia illucens is a cosmopolitan fly heavily used by industrial companies to reduce biowaste and produce protein and fat for poultry and aquaculture feed. However, the natural history and the genetic diversity of the BSF are poorly known.

\section{Results}

In this study, we analyzed 677 C01 sequences derived from samples found all over the five continents, leading us to discover 52 haplotypes, including ten major haplotypes. We refined the definition of these haplotypes by sequencing 59 mitochondrial genomes. We could derive an estimate of the separation events of the different haplotypes at more than two million years for the oldest branches. This worldwide cryptic genetic and genomic diversity is mirrored at a local scale in France, in which we found five major haplotypes sometimes in sympatry.

\section{Conclusions}

Our data resolve the phylogenetic relationships between the major lineages and give insights into the dispersal and the numbers of BSF neo-introduction at global and local scales. Our results indicate that commercial BSF stock's genetic and genomic diversity is very low. In addition, this broodstock participates in disseminating the BSF in the wild. Taken together, these results call for a better understanding of the genomic diversity of the BSF to unravel possible specific adaptations of the different lineages for industrial needs and to initiate the selection process.

\section{Background}

The black soldier fly (BSF) Hermetia illucens (Linnaeus, 1758) is a Diptera of the Stratiomyidae family. It probably originated from Mexico, then spread to the south of the USA and Latin America over the last thousand years ${ }^{1}$. These geographical areas are now considered their natural area ${ }^{1,2}$. The BSF is now a cosmopolitan fly found in tropical, subtropical, and tempered regions, from the 40th parallel North and the 45th South 2, 3, where it is considered an exotic, non-invasive species ${ }^{4,5}$. This fly likely spread through shipping routes ${ }^{6}$. While studies based on naturalistic observations show that it was first discovered in Malta in $1926^{7}$, it has been suggested that it was introduced in Italy about 500 years ago ${ }^{8}$. The BSF appeared in the early 1950 s in the South-East of France ${ }^{9}$ before spreading through the South-West ${ }^{10}$ along the Rhône valley ${ }^{11}$ and finally along the Atlantic coast ${ }^{12}$. Currently, the BSF can be found on the whole European territory, with few exceptions. It is reasonable to assume that the rise of international traffic and the industrial use of the BSF may have led to a steady flow of introductions in France and worldwide ${ }^{13,14}$.

The BSF was initially used in forensic entomology to date the post-mortem time ${ }^{15}$. Industrial companies heavily use it to reduce biowastes ${ }^{16,17}$, to produce protein $17,18,19$, and fat $17,18,20$. These products are used to make animal food ${ }^{21}$ or biodiesel 22,23 .

BSF industries face similar issues as other farms: knowing how to master their model's biological and genetic aspects to get the best out of it. To this end, it is crucial to know the genetic diversity of BSF existing internationally, nationally, and locally. Many articles exist about the nutritional aspect of the BSF $20,24,25$, 26,27 or its microbiota $28,29,30$, few have focused on the genetic diversity of the BSF. We have some evidence on the extent of BSF genetic diversity from previous studies. Ten haplotypes based on the $\mathrm{CO} 1$ gene were found in South Korea with 245 individuals ${ }^{31}$. Still, on the C01 gene and a worldwide sampling, 56 haplotypes were found with a divergence rate up to $4.9 \%$, and it has been shown that individuals belonging to divergent lineages are still interfertile 32 . Finally, based on 15 microsatellite markers, 16 genetic clusters were found with hot spots in South America ${ }^{33}$. Other works focused on more fundamental aspects of BSF and assembled a reference mitochondrial genome and a chromosomal-scale nuclear genome ${ }^{34,35,36}$.

Within the framework of the BSF industry, phylogeography can allow on the one side to account for genetic diversity at different scales (global, national, and local) and, on the other side, to have keys on the geographical origin of the species present in a territory or used by companies. This study may suggest local adaptations or some phenotypic plasticity due to habituation to different biomes.

In this study, we quantified this diversity at different scales: worldwide by studying the variation within the CO1 gene, and more locally at the level of France. We also analyzed the role of livestock BSF farming in the diversity present on the French territory. Our report is based on data obtained by sequencing 113 individuals for the $\mathrm{CO} 1$ gene and mining $564 \mathrm{CO} 1$ sequences. We also sequenced and assembled 59 mitochondrial genomes. We also show the great and hidden genetic diversity within Hermetia illucens at all observation scales, focusing specifically on France ( 90 French individuals were analyzed, allowing us to find nine different haplotypes). In addition, we are also providing here date of the separation events of the different haplotypes found. This separation turns out to be more than two million years old for the most distant haplotypes.

\section{Materials And Methods}

\section{Sampling}

We decided to conduct a global sampling followed by a specific focus on France. A detailed table of samples and associated metadata is provided in (Table S01 - supplementary). The origin of all $\mathrm{CO} 1$ sequences used in our study is summarized in Table 1, and a summary of sample numbers by continent is provided in Table 2. Samples were collected during summer 2020 and stored in $100 \%$ ethanol at $-20^{\circ} \mathrm{C}$ prior to DNA extraction. In France, the collection was done with the help of the compost citizen network ${ }^{37}$. The larvae came exclusively from compost and the adults collected were close to the composting area. 
Between one and ten individuals (larvae or adults) were collected per area. In some French localities, collections in the wild have been made near BSF breeding areas (about five kilometers). For the other parts of the world, the individuals came either from compost or breeding farms.

In SRA, we gathered all the CO1 sequences on NCBI and Bold and whole-genome sequencing data and reconstructed the mitochondrial genome. In France, sampling was carried out during the summer of 2020 in shared and personal compost sets. We obtained larvae in the areas of Blois, Bordeaux, Toulouse, Montpellier, Lyon, Paris. In Africa, individuals from compost or livestock farms were collected during 2019. In Latin America, larvae from French Guiana compost were collected during summer 2019. An adult was found in 2016 in Mexico. In Asia, individuals collected from Taiwan either from a breeding farm or compost. These were mainly L5 larvae and pupae; some adults were also recovered. We were surprised in the Bayonne area to have BSF-like larvae, which turned out to be Exaireta spinigera larvae.

\section{DNA extraction}

We extracted DNA from larvae, pupae, and imago under the same conditions by grinding individuals in liquid nitrogen using a mortar and pestle. The ground samples were then processed according to the protocol of the Macherey-Nagel AXG $100^{38}$ kit for the isolation of genomic DNA from tissue with some modification: we added a second purification step with $3.5 \mathrm{ml}$ of freshly prepared ethanol and centrifugation at $15,000 \mathrm{rpm}$ at $4{ }^{\circ} \mathrm{C}$ for $15 \mathrm{~min}$ just before the final re-suspension. The DNA pellet was finally re-suspended in $100 \mu \mathrm{L}$ of Tris $10 \mathrm{mM}$ EDTA $1 \mathrm{mM} \mathrm{pH} \mathrm{8.0.} \mathrm{The} \mathrm{purity} \mathrm{was} \mathrm{evaluated} \mathrm{by} \mathrm{spectrophotometry}$ using a Nanodrop ${ }^{39}$, and the concentration was quantified using the Qubit kit DNA Broad range ${ }^{40}$. DNA integrity was monitored using routine agarose gel electrophoresis.

\section{C01 Sequencing}

We designed specific $\mathrm{CO} 1$ primers from the reference mitochondrial DNA ${ }^{34}$ (Table S02 - supplementary), then a mix was realized using NEB $5 \mathrm{X}$ standard buffer One Taq. The mix is then amplified in ONETAQ HOT 45 PCR. We purified the PCR products using the Illustra Exostar 1-step GE Healthcare kit before Sanger cycle sequencing. Cycle sequencing reactions were performed using the BigDye 3.1 chemistry, purified by precipitation, and sequenced using an $\mathrm{ABI}$ 3130 sequencer (Applied Biosystems). Base-calling was performed using the sequencing analysis software version 5. 3 (Applied Biosystems). We assembled forward and reverse reads using the Geneious V R $11^{41}$ assembler with the sensitivity set at medium/fast. The regions containing the primers were excised, and we kept only the sequences having more than $75 \%$ of high-quality base calls.

\section{Multiple sequence alignment and haplotype network reconstruction}

We aligned $\mathrm{CO} 1$ sequences obtained in the laboratory and those recovered from the databases using the MAFFT ${ }^{42}$ aligner included as a plug-in in the Geneious software ${ }^{41}$. We used the algorithm G-INS-i with a scoring matrix 200 PAM / k=2, a gap open penalty at 1.53 , and an offset value at 0.123 . We exported the multiple sequence alignment in nexus format ${ }^{43}$ for haplotype network reconstruction and visualization using POPART ${ }^{44}$. We modified the output file to add geographical origins to the sequences.

\section{Shotgun sequencing and mitochondrial DNA assembly}

A total of 56 BSF individuals were used for whole-genome shotgun sequencing using an Illumina method by Novogene UK $45,46,47,48,49$. The technique used is pair-end with a coverage rate of $4,68 \mathrm{X}(\mathrm{SD} 0,50)$. The resulting sequences were checked for quality and counted for read parity. We take advantage of the fact that mtDNA is highly repeated in insect DNA. In insects, mtDNA represents on average $0.42 \%$ of the total DNA in the genome sequence project ${ }^{50}$. As stipulated, we used $5000 \mathrm{~kb}$ of data/sample $(4-5 \mathrm{x}$ coverage) for the mitochondrial assembly, which on average contains $(5000 * 0,42) / 100=21000 \mathrm{~kb}$ of mtDNA. As the mitochondrial genome is about $15 \mathrm{~kb}$, we obtain coverage of $1400 \mathrm{x}$ for this genome which is largely sufficient to obtain a complete genome in one contig. The sequences were assembled de novo with the MEGAhit software ${ }^{51}$ (based options); the mitochondrial genomes were thus recovered from the assemblies by blast ${ }^{52}$ using the reference BSF mitochondrial genome sequence (NC_035232). THE MITOCHONDRIAL DNAS WERE ANNOTATED using MITOS online software ${ }^{53}$, and the D-Loop zone was removed. We added four complete mitochondrial sequences from databases or reconstructed via reading sequences available on the SRA ${ }^{54}$ using the same assembly procedure and then aligned under the same conditions as previously mentioned.

\section{C01 and whole mitochondrial genome phylogeny}

We use the MEGAX ${ }^{55}$ software to determine the best model and compute an ML Tree with 500 Bootstraps using a complete deletion parameter. We visualized the data with the online tool ITOL ${ }^{56}$, and we rooted the tree with the Stratiomyidae Exaireta spinigera. We chose Exaireta spinigera because it was the closest species for which we have a complete mitochondrial genome (the blastn result of the complete mitochondrial genome of Hermetia illucens vs. Exaireta spinigera gives us $81.28 \%$ of similarity). To simplify the interpretation of the phylogeny, we have kept only a single identical sequence per haplotype.

\section{Time tree}

We used the BEAST software ${ }^{57}$ to estimate the divergence time of the different BSF groups. The analysis was performed with an expansion growth model and an uncorrelated lognormal relaxed clock with the proposed insect molecular clock. Ten million iterations were performed, and then with the TreeAnotator ${ }^{58}$, the obtained trees were clustered with a burn of $25 \%$ of the trees. The visualization of the final tree was done with the online software ITOL ${ }^{56}$.

\section{Results}




\section{Haplotype Network}

We reconstructed a haplotype network from the analysis of 677 sequences spanning 658 nucleotides of the C01 mitochondrial gene. A total of 52 distinct haplotypes were delineated from the analysis of this $\mathrm{CO} 1$ fragment of one to 230 fly samples (Figure 1).

We found 73 segregating sites with a Tajima-D of 45.1413, suggesting a lack of rare alleles, which could come from a contraction of the population.

There were 31 haplotypes made of a single sequence. We observed 11 haplotypes made of between two and four sequences derived from the same continent; that total of 42 haplotypes will be referred to as minor haplotypes. We observed ten haplotypes made of between five and 230 sequences, including six multicontinental haplotypes that we will refer to as major haplotypes. In conclusion, we found an important diversity both within and between continents using the C01 phylogenetic mitochondrial gene. Minor haplotypes represent $8.5 \%$ of all sequences (58 sequences) and $80.8 \%$ of haplotypes. Major haplotypes represent $91.5 \%$ of the sequences (619 sequences) and $19.2 \%$ of the haplotypes.

In Europe, we found ten distinct haplotypes from 118 sequences, nine for Asia from 277 sequences, five for Oceania from 106 sequences, 30 for South America from 55 sequences, four in Africa from 78 sequences, and seven in North America from 43 sequences. Some major haplotypes are represented in a more restricted area. For example, haplotype B is mainly present in Asia (figure 1). We observed a high haplotype diversity in South America with 28 minor haplotypes that contain between one and four sequences. The haplotype $\mathrm{C}$ contains all the sequences derived from farmed strains of the industry working on BSF.

\section{Global distribution analysis}

We visualized the actual distribution of the different haplotypes found previously on a distribution map (figure 2).

Each haplotype from $\mathrm{A}$ to $\mathrm{J}$ is now represented with a color; haplotypes that are numbered in the haplotype network (figure 1) are now represented in grey.

We observed great variability in South America highlighted on the map by the grey pie charts representing mostly minor haplotypes. We observe 22 singletons in South America out of the 52 haplotypes found, more than $40 \%$ of the total diversity. On the other hand, we found six out of ten major haplotypes in Europe.

Haplotype A and C were the most represented haplotypes in our sampling: Haplotype A was present on all continents and represented $34 \%$ of all sequences, and haplotype $\mathrm{C}$ was only absent from South America and represented $18 \%$ of all sequences. Some haplotypes seemed to be restricted to some world areas, such as haplotype G, which was present in all the localities in West Africa and surprisingly in a Portuguese sample, or the haplotype $\mathrm{H}$ in South America. Haplotype F was present in Europe, Uganda, and South Korea. Haplotype E was more present in Asia and Oceania as well as in North America. Finally, some haplotypes such as $\mathrm{B}, \mathrm{I}$, and $\mathrm{J}$ present rare sequences found in more restricted areas such as $\mathrm{B}$ in Korea or $\mathrm{H}$ and $\mathrm{J}$ in France (figure 2).

\section{C01 Phylogeny}

During our $\mathrm{CO} 1$ gene analyses, some BSF-like larvae were identified as Exaireta spinigera larvae. The sequences were amplified and sequenced with the same primers as for BSF.

By taking only one sequence for each haplotype, we have reconstructed a phylogeny of the C01 gene sequence. The CO1 phylogeny was rooted with the Exaireta spinigera (figure 3). A sample from Cameroon was revealed to belong to a closely unidentified species of BSF and was termed Hermetia $s p$. Cameroon in our subsequent analysis (Blastn score between Hermetia sp. from Cameroon and BSF gives us $85.01 \%$ identity with an E Value of 2e-174).

The $\mathrm{CO} 1$ gene phylogeny supports the analyses performed with the haplotype network (Figure 1), showing remarkable sequence diversity among the C01 gene. Interestingly, all the commercial strains are found in the $\mathrm{C}$ haplotype (a detailed phylogenetic tree is given as a supplementary with an asterisk placed on individuals from farms (Figure S03 - Supplementary).

A group containing haplotypes 35 to 42 and haplotypes B and C forms a divergent group from the others, with strong statistical support (bootstrap value of 0.99). Some individuals captured in the wild have been found near BSF commercial breeding sites. For example, we have captured $\mathrm{C}$ haplotype individuals in Avignon close by companies working with the BSF.

Although the global resolution of the deep branches of the tree is low, it is also possible to better understand the relationship between the haplotypes: haplotype B and C are close to each other and well separated from other haplotypes. The other haplotypes are not very well resolved with the CO1 sequences. Finally, the samples coming from Latin America are scattered all over the tree, showing their great diversity and supporting the hypothesis of the geographical origin of the BSF.

\section{The global BSF diversity is mirrored at the local level in France}

Among the ten major haplotypes, our sampling effort in France showed that five are present in France in addition to a unique haplotype only found in France (Figure 4) in La Rochelle. Haplotype A remains the dominant haplotype in France (71.1\% of all sequences) in seven of the eight localities (excluding Beaufort en Vallée, where the flies come from a company). Regarding the haplotype C, BSF belonging to this group have been found in four locations in the regions of Poitiers, Bordeaux, Beaufort-En-Vallée and Montpellier. Except for Beaufort-En-Vallée, where the flies come from a company, the other BSF come from natural environments. BSF industries exist in all these localities. More surprisingly, different haplotypes coexist in some identical localities. For example, we found three different haplotypes in the region of Paris and four in the region of Montpellier. Finally, we found a singular haplotype in Colomiers ( $\mathrm{J}$ ) and La Rochelle (1). 


\section{Complete mitochondrial genome analyses}

To better understand BSF phylogeny and estimate the timing of haplotype divergence, we reconstructed and analyzed the mitochondrial genomes of 60 individuals, including 3 Exaireta spinigera (used as an outgroup). The 60 reconstructed mitochondrial genomes have the typical structure of insects mtDNA: 13 protein-coding genes, 22 tRNA, two rRNA-coding genes, and non-coding regions and control regions (an example is given as a supplementary (Figure S04 Supplementary). The genetic differences are homogeneously distributed overall mtDNA and are not localized on specific genes (Figure 5A). A great genetic diversity at the mitochondrial genome level is observed. Two very homogeneous groups, $A$ and $C$, are well represented, and sequences of the $C$ haplotype are wildly divergent all along the genomes from the others. The two sequences with the most differences are 55-F-Angouleme and 21-Ghana, with a similarity percentage of $96.373 \%$ (all results are in supplementary (Figure S05 - Supplementary).

We also wanted to know if the mutations are equally (or not) distributed between the different genes. Therefore, we compared the level of similarity of the 13 mitochondrial genes of the $57 \mathrm{BSFs}$ (fig 5B) to those of Exaireta spinigera. The results are presented as percent sequence similarity.

We grouped the individuals into three different categories:

-Haplotype A (Figure 5 B1), all genes show the same differences with those of Exaireta spinigera; only the NAD6 gene shows slight differences.

Haplotype C (Figure 5 B2) shows that most of the mitochondrial genes are identical except for the ATP8 gene, which is different for four individuals (11 Angouleme; 14 Kenya; 19 Kenya, and 15 Kenya). Slight differences are also present in the NAD3 and NAD6 genes.

-Haplotype D-F-G-H-I (Figure 5 B3), the profiles are slightly different, with a more substantial disparity for the ATP8, NAD1, and NAD5 genes, especially the Mexican one (57). The profiles show the same pattern except for the ATP8 gene of the individual from Mexico (57), which is more conserved.

\section{Complete mitochondrial genome phylogeny}

The complete mitochondrial genome phylogeny demonstrates the very high genetic divergence among BSF populations found with the CO1 gene analysis (Fig $6 \mathrm{~A})$. The increased sensitivity of the analysis, due to the size of the sequences (13.96 kb, 13 genes), robustly resolves the relationships between different haplotypes identified previously (nodes $>95 \%$ ). In addition, it allows us to distinguish subgroups within the previously determined haplotypes. Two major divisions emerge within the tree: group $\mathrm{C}$ (which includes all commercial strains) and others. Two subgroups emerge within the $\mathrm{C}$ group (11 - $\mathrm{F}-\mathrm{Angouleme}$ 14 Kenya; 19 Kenya; 15 Kenya and the others).

The other large group contains the sequences of the A D I G and $\mathrm{H}$ haplotypes. The I G and $\mathrm{H}$ haplotypes are related and close to each other. The very cosmopolitan A haplotype is well conserved with limited mitochondrial sequence divergences.

Moreover, although phylogenetically close, the I G and $\mathrm{H}$ haplotypes are found in very disparate areas (the $\mathrm{G}$ haplotype is mainly in Africa, the I in Europe, and the $\mathrm{H}$ in Latin America), supporting multiple worldwide introductions.

Strikingly the high level of the conservation of the ATP8 sequence described above in the Mexican sample (haplotype D) does not explain the basal position of the sequence in the phylogeny; indeed, we tested the phylogeny by removing the ATP8 gene, and no change was found (Figure S06 - Supplementary).

The ATP8 gene alone is not sufficient to explain the appearance of the observed subgroups. However, unlike the CO1 gene, which varies only slightly within this haplotype, the NAD2, NAD3, NAD5, and NAD6 genes are more variable.

The average percentage of identity between haplotypes $A$ and $C$ is about $96.39 \%$. Between haplotype $\mathrm{C}$ and $\mathrm{D} 96,54 \%$. Between haplotype $\mathrm{C}$ and $\mathrm{H} 96.40 \%$ and 96.43\% between C and I. Between A and D 97.82\% (all results are in Figure S03 - Supplementary).

\section{The divergence time of the different groups}

The time tree (fig 6B) gives us information about the divergence time of the different mitochondrial genomes. We obtained identical Bayesian and ML trees. We noted a relatively long divergence time between haplotype $C$ and the others ( 2.2 million years). The others have diverged more recently (about 1.2 million years). Within the $C$ haplotype, a divergence has been about 300.000 years, while in the other groups, the divergence times are much lower, about 20.000 years for the A haplotype.

The time of divergence with Exaireta spinigera has been estimated at 110 million years, confirmed by the literature that gives us 112 million years with the Timetree tool ${ }^{59}$.

\section{Discussion}

Hermetia illucens is now considered a species of major interest for the food industry ${ }^{60}$. Thus, it became urgent to study the genetic diversity within the different populations at different scales. Analyzing the different haplotypes in the same geographical area is a powerful tool to study the recent history of BSF. An in-depth study of the genetic variations between the different haplotypes may allow us to resolve better the scenario of the arrival of BSF in a territory.

In this study, we uncovered a high genetic variability of mitogenomes within the species Hermetia illucens. We found that haplotype $\mathrm{C}$ is the most divergent. The dating of the separation events with the other haplotypes goes back more than 2 million years. We found a well-resolved separation within haplotype $\mathrm{C}$ $(>95 \%)$; the other haplotypes are also well resolved $(>95 \%)$. 
Our study confirms the genetic diversity found in previous papers ${ }^{31,32,33}$. We found a comparable number of genetic clusters, although we added many more sequences of diverse origin. For example, Ståhls et al. ${ }^{32}$ found 56 distinct haplotypes with $418 \mathrm{CO} 1$ sequences, and we found a comparable number of haplotypes based on the analysis of $677 \mathrm{CO}$ sequences. These results suggest that we have captured a major part of the extant BSF worldwide diversity. However, in South America, we found 30 haplotypes among the 55 sequences sampled that open up the possibility of a still unknown genetic diversity in this part of the world.

With the most remarkable genetic diversity being found in South America, the hypothesis of the geographical origin of BSF in Latin America is further confirmed. Apart from this continent of origin, there seem to be many neo-introductions on all continents with divergent mitochondrial lineages. The analysis of complete mitogenomes supports the hypothesis of several distinct introductions in Europe and other continents. Our results contradict the scenario of Kaya et al. ${ }^{33}$ based on microsatellites for a single BSF introduction in Asia and Australia. Indeed, our data support the presence of highly divergent major haplotypes A, C, and E in Australia and major haplotypes A, B, C in Asia, in addition to several orphan haplotypes. As the divergence times between these major haplotypes is estimated at around two million years, this pattern is incompatible with a single introduction by man in the recent period but supports multiple and independent introductions.

In the same way, Kaya et al. ${ }^{33}$ propose at least three distinct introductions in Europe. Our data considerably increases this number: at least seven different haplotypes are found ( $A, I, J, G, F, C$, and E), supporting at least seven distinct introductions. Similarly, at least four introductions in Africa arose. By increasing the sample size and using more resolutive markers, we obtained a more precise description of BSF genetic diversity and better understood its natural history. However, due to the frequent, multiple, and probably ongoing introductions worldwide of the BSF, understanding the detailed introduction history might be challenging.

Even at a national scale, the BSF genetic diversity is vast. By taking the example of France, we were able to show that the diversity observed at the global scale is also found at the national scale (five of the ten major haplotypes found worldwide). Moreover, we have haplotype mixtures at the local level, confirming the observations on microsatellite data ${ }^{33}$. For example, in the region of Paris, we have a mix of three haplotypes (A, F, and I), and in the region of Montpellier, a mix of four haplotypes (A, B, C, and E) - Figure 4.

France contains on its territory a great diversity of haplotypes, whose origin can be explained either by the historical introduction of the year $1950{ }^{9}$ in addition to more recent neo-introductions. The haplotype A, which is by far the most common haplotype in wild BSF found in France (71.1\%) and in Europe, may correspond to the initial introduction. However, it is difficult to understand the exact scenario of the introduction of BSF in France. For example, the divergence times within French flies belonging to haplotype A (20.000 years) tend to prove the recent but multiple introductions in France of flies belonging to this group rather than a single introduction by men followed by diversification on the territory. Our data support a complex scenario involving multiple and repetitive introductions of the BSF from closely related (same phylogenetic groups) and distantly related (divergent phylogenetic groups) individuals in France. A possible scenario involves introducing the haplotype $A$ in the $1950 \mathrm{~s}^{9}$, which spread gradually on the territory. More recent introductions arose explaining the occurrence of the haplotypes $\mathrm{J}, \mathrm{G}$, and I. Finally, introductions related to the industrial activity of the BSF of the haplotype C arose.

Jefferey Tomberlin, a pioneer in the field, also indicates in a recent article on the structure and demography of the BSF based on microsatellite data ${ }^{33}$ that the captive populations used for breeding in Europe and North America derive from a common, genetically related strain. Our analysis based on CO1 and complete mitochondrial genome sequences confirms this result: the haplotype that we have named $\mathrm{C}$ (for commercial) contains all the sequences of industrial origin with very few genetic diversity. Interestingly, this commercial group does not have any South American relatives, but Kayla et al. report wild North American populations related to the haplotype $C$ (but not similar). This result suggests a possible North American origin for the BSF commercial stock. Our data support a possible alternative scenario because both network haplotypes and C01 phylogeny indicate that the C haplotype is closely related to the $\mathrm{B}$ haplotypes and eight other haplotypes distributed in Asia and Europe. However, the exact origin of the commercial stocks might be difficult to decipher due to the naturalization of the $\mathrm{C}$ haplotypes in many locations.

Indeed, specific individuals collected in the wild and belonging to this group were collected in the vicinity (around $5 \mathrm{~km}$ ) of industries breeding BSF. For example, if we focus on the commercial haplotype analysis in France (the $\mathrm{C}$ haplotype), the wild BSF belonging to the haplotype $\mathrm{C}$ may have escaped from industries in the regions of Poitiers, Bordeaux, Avignon, and Montpellier. This last point raises the question of the biosecurity of BSF farms around the world. Indeed, suppose the industries carrying outbreeding of BSF do not opt for optimal safety. In that case, the escape of BSF of industrial origin can lead either to establishing the BSF in territories where it was not yet present or to a replacement-hybridization of the local populations by the industrial strain. This would lead inexorably to a loss of the local genetic diversity, thus potentially erasing some local adaptation to specific environmental conditions.

However, it is interesting to note that in Ståhls et al. $2020^{32}$, in contrast to Kaya et al. $2021^{33}$ and our study, some genetic diversity in the breeding strains in Europe and North America have been evidenced (10 haplotypes). This may be due to a broader sampling effort of commercial strains. Indeed, Stahls ${ }^{32}$ had the opportunity to sample many small farms leading to 292 BSF sequences from rearing cultures. This allowed them to access a broader genetic diversity not found in our study and Kaya et al. ${ }^{33}$ It is also possible that smaller farms did not obtain BSF from the dealers who constituted most of the more prominent industries but directly from in Natura acquisitions. Nevertheless, our results confirm that the industrial actors work with the same and unique haplotype.

We also evidence that the mitochondrial genome is perfectly suited to differentiate the genetic diversity and resolve phylogenetic relationships. It allows us to date the natural history of the BSF, bringing a sum of details that were impossible to distinguish based only on the C01. For example, we report puzzling variations in the level of conservation of the ATP8 genes for some samples (haplotypes C and D). Interestingly, these variations have already been found in other insect species (for example, in Coleoptera, see Zhang et al. $2019{ }^{60}$, and in grasshoppers, see Li et al. $2018{ }^{61}$ ). The ATP8 gene is small (160 bp on average in insects) and encodes for a subunit of the complex V of the ATP synthase. In Hermetia illucens, the initiator codon of the ATP8 gene is ATT, is 167 
bp long, and it overlaps with the ATP6 gene. It has been assumed in other species that the modification of the sequence of the ATP8 gene can affect the structure of mitochondrial complex $\mathrm{V}$ and thus its function.

Furthermore, it has been shown that changes within ATP synthase can be explained by adaptation to different ecological environments ${ }^{62,63,64}$. This indicates that some local adaptation driven by the ATP8 genes may exist in BSF, as it has already been shown in other insects. This can lead to an adaptation to survive in high-altitude environments, i.e., at a lower oxygen level and low temperature ${ }^{65}$. Even if these variations did not change the phylogeny of the BSF, it could be a clue to some local adaptation signal.

Complete mitochondrial genome data allows us to make a robust and well-resolved time tree of the species. The divergence times between the different haplotypes are surprisingly long (more than two million years for the most divergent lineages), and the differences observed between the sequences prove that there is still unsuspected and hidden genomic diversity for the species. Comparing it to Drosophila, two million years correspond to many speciation events leading to different reproductively isolated species ${ }^{66}$. By contrast, Stahls et al ${ }^{32}$. indicated that BSF belonging to phylogenetically divergent haplotypes can still cross and generate offspring, indicating no apparent reproductive isolation in laboratory conditions. Interestingly, our data evidence that the cohabitation of different haplotypes at the same locality could lead to crosses between haplotypes. Further investigations are thus needed to understand the possible genetic barriers between highly divergent BSF groups but also the possible patterns of introgression in natural BSF populations. This phenomenon might considerably confuse the elucidation of the population structure and the natural history of these species.

Finally, it is interesting to recall that, if indeed, most industrial actors use the same haplotype for the industry, the other haplotypes present in France are wildly divergent from this one. Strikingly, we found in one compost bin in the region of Paris flies belonging to two different haplotypes (A and F). Thus, crossbreeding between these two individuals belonging to these two haplotypes would bring a solid genetic mix that could benefit breeding. Associated with this significant genetic diversity, BSF may also have some significant phenotypic diversity that might be useful for specific industrial usage and selection processes to increase performances or ameliorate peculiar traits or behaviors ${ }^{67}$.

\section{Conclusion}

Through the analysis of 60 mitochondrial genomes during this study, we proposed a robust and well-resolved time tree of the BSF that confirms the vast and hidden genetic diversity within Hermetia illucens. Our result elucidates the phylogenetic relationship and time divergence between the different BSF lineages and provides a plausible scenario for BSF expansion in the world via multiple and independent introductions. With $677 \mathrm{COI}$ gene sequences coming from a broad geographical sample, we highlighted the genetic diversity at the level of the different continents evidencing recurrent and multiple introductions outside the geographical area of origin in Latin America. However, the exact scenario of introductions, even at a national scale in France, is still challenging to understand due to probable multiple and independent introductions and possible interbreeding between different populations that coexist in the same geographical area. We have also shown that many BSF industrial companies work with flies that derive from a unique population. We would also like to remind that the apparent biosecurity deficiency within some BSF industrial companies probably led to the escapement and possible local introduction/replacement of natural populations of BSF. Finally, our data indicated that it becomes extremely interesting to take a closer look at the nuclear genomes' evolution of the BSF to understand better the natural history of the BSF as well as specific adaptations of the different lineages that might be useful for industrial needs and to initiate the selection process for peculiar traits.

\section{Declarations}

\section{Ethics approval and consent to participate}

Not applicable

\section{Consent for publication}

Not Applicable

\section{Availability of data and materials}

The dataset generated during the study has been deposited in the European Nucleotide Archive database under Accession Numbers PRJEB48031 Bioprojet. Alignment and raw data can be downloaded following this link http://gofile.me/2ppPR/QWjM33T7y the supplementary can be downloaded following this link: http://gofile.me/2ppPR/rwaGSXDQU

\section{Competing interests}

The private company Cycle Farms partially granted this project, but at any moment, the company has influenced the design and the analysis of the results nor the writing of the manuscript and its conclusions. No commercial products have been developed based on the outputs of this article. None of the authors have any competing interests.

\section{Funding}


This work was funded in the framework of a partnership between the CNRS and the CycleFarms company. JG is supported by a CIFRE Ph.D. Grant from the Agence Nationale pour la Recherche et la Technologie (ANRT)

\section{Author's Contributions}

JG carried out the sampling, performed data curation, and wrote the original draft. NP performed data curation and reviewed the manuscript. GB helped with the data curation and reviewed the manuscript. JF conceptualized and supervised the study, performed data curation, reviewed and finalized the manuscript.

\section{Acknowledgments}

We would like to thank Floran Laville and Frederic Marion-Poll for their continuous support and help during this project. We warmly thank the members of the Réseau Compost Citoyen for their precious help during the sampling in France. We also thank Matan Shelomi (National Taiwan University), Meng-Kun Wu, Shu-Min Chen, Jing-Jiun Huang (Yi Mi Community College, Taiwan) for flies from Taiwan. We thank the International Centre of Insect Physiology and Ecology (ICIPE) from Nairobi City, Kenya. We thank Mathieu Chouteau from CNRS LEESIA, in French Guiana. We finally thank Benoit Gilles for his help at the beginning of this project.

\section{References}

1. Hauser, Martin, and Marshall Woodley. "The Historical Spread of the Black Soldier Fly, Hermetia Illucens (L.) (Diptera, Stratiomyidae, Hermetiinae), and Its Establishment in Canada." Journal of the Kansas Entomological Society 146 (December 1, 2015): 51-54.

2. Rozkosný, R. A Biosystematic Study of the European Stratiomyidae (Diptera): Volume 2 - Clitellariinae, Hermediinae, Pachygasterinae, and Bibliography. Series Entomologica. Springer Netherlands, 1983. https://www.springer.com/gp/book/9789061931355.

3. Mason, Franco, Rudolf Rozkošný, and Martin Hauser. "A Review of the Soldier Flies (Diptera: Stratiomyidae) of Sardinia." Zootaxa 2318, no. 1 (December 22, 2009): 507-30. https://doi.org/10.11646/zootaxa.2318.1.20.

4. https://tinyurl.com/4f6nbe2t

5. Wang, Yu-Shiang, and Matan Shelomi. "Review of Black Soldier Fly (Hermetia Illucens) as Animal Feed and Human Food." Foods (Basel, Switzerland) 6, no. 10 (October 18, 2017). https://doi.org/10.3390/foods6100091.

6. Picker, Mike, Charles Griffiths, and Alan Weaving. Field Guide to Insects of South Africa. Penguin Random House South Africa, 2002.

7. Lindner, Erwin. Die amerikanische Hermetia illucens L. im Mittelmeergebiet: (Stratiomyiidae, Dipt.). Akadem. Verlagsges., 1936.

8. Benelli, Giovanni, Angelo Canale, Alfio Raspi, and Gino Fornaciari. "The Death Scenario of an Italian Renaissance Princess Can Shed Light on a Zoological Dilemma: Did the Black Soldier Fly Reach Europe with Columbus?” Journal of Archaeological Science 49 (September 1, 2014): $203-5$.

https://doi.org/10.1016/j.jas.2014.05.015.

9. Barbier, Jean. "Introduction en France d’un Diptère Stratiomyide américain." Bulletin de la Société entomologique de France 57, no. 7 (1952): $108-108$.

10. Dauphin, Patrick. "Présence de Hermetia illucens (LINNÉ, 1758) dans le sud- ouest de la France (Diptera Stratiomyiidae)," n.d., 3.

https://www.researchgate.net/publication/313631958_Presence_de_Hermetia_illucens_Linne_1758_dans_le_sudouest_de_la_France_Diptera_Stratiomyiid

11. Richoux Philippe. "Sur La Présence d'Hermetia Illucens(Linnaeus, 1758) (Diptère Stratiomyidae) Dans La Région Lyonnaise/Presence of Hermetia Illucens (Linnaeus, 1758) (Diptera Stratiomyidae) in the Region of Lyons." Bulletin Mensuel de La Société Linnéenne de Lyon 78 (May 1, 2009 ): 137-38.

https://doi.org/10.3406/linly.2009.13722.

12. Maquart Pierre-Olivier, Richard Denis, and Jesse Willems. "First Record of the Black Soldier Fly, Hermetia Illucens, in the Western Regions of France (Vendée, Loire-Atlantique, Ille-et-Vilaine) with Notes on Its Worldwide Repartition (Diptera, Stratiomyidae)." Bulletin de La Société Entomologique de France 125 (March 20, 2020): 13-18. https://doi.org/10.32475/bsef_2104.

13. Leclercq, M. "Dispersion and transport of noxious insects: Hermetia illucens L.(Diptera Stratiomyidae)." Bulletin des recherches agronomiques de Gembloux, 1969. https://scholar.google.com/scholar_lookup?

title=Dispersion+and+transport+of+noxious+insects\%3A+Hermetia+illucens+L.\%28Diptera+Stratiomyidae\%29\&author=Leclercq\%2C+M.\&publication_year

14. Sheppard Craig, D., G. Larry Newton, Sidney A. Thompson, and Stan Savage. "A Value Added Manure Management System Using the Black Soldier Fly." Bioresource Technology 50, no. 3 (January 1, 1994): 275-79. https://doi.org/10.1016/0960-8524(94)90102-3.

15. Lord, W. D., M. L. Goff, T. R. Adkins, and N. H. Haskell. "The Black Soldier Fly Hermetia Illucens (Diptera: Stratiomyidae) As a Potential Measure of Human Postmortem Interval: Observations and Case Histories." Journal of Forensic Science 39, no. 1 (January 1, 1994): $215-22$.

https://doi.org/10.1520/JFS13587J.

16. Gold, Moritz, Jeffery K. Tomberlin, Stefan Diener, Christian Zurbrügg, and Alexander Mathys. "Decomposition of Biowaste Macronutrients, Microbes, and Chemicals in Black Soldier Fly Larval Treatment: A Review." Waste Management (New York, N.Y.) 82 (December 2018): 302-18.

https://doi.org/10.1016/j.wasman.2018.10.022.

17. Hoc, B., M. Genva, M.-L. Fauconnier, G. Lognay, F. Francis, and R. Caparros Megido. "About Lipid Metabolism in Hermetia Illucens (L. 1758): On the Origin of Fatty Acids in Prepupae." Scientific Reports 10, no. 1 (July 17, 2020): 11916. https://doi.org/10.1038/s41598-020-68784-8.

18. Ewald, Nils, Aleksandar Vidakovic, Markus Langeland, Anders Kiessling, Sabine Sampels, and Cecilia Lalander. "Fatty Acid Composition of Black Soldier Fly Larvae (Hermetia Illucens) - Possibilities and Limitations for Modification through Diet." Waste Management (New York, N.Y.) 102 (February 1, 2020): 40-47. https://doi.org/10.1016/j.wasman.2019.10.014. 
19. Giannetto, Alessia, Sabrina Oliva, Carlos Frederico Ceccon Lanes, Fabio de Araújo Pedron, Domenico Savastano, Cosimo Baviera, Vincenzo Parrino, et al. "Hermetia Illucens (Diptera: Stratiomydae) Larvae and Prepupae: Biomass Production, Fatty Acid Profile and Expression of Key Genes Involved in Lipid Metabolism." Journal of Biotechnology, October 31, 2019. https://doi.org/10.1016/j.jbiotec.2019.10.015.

20. Spranghers, Thomas, Matteo Ottoboni, Cindy Klootwijk, Anneke Ovyn, Stefaan Deboosere, Bruno De Meulenaer, Joris Michiels, Mia Eeckhout, Patrick De Clercq, and Stefaan De Smet. "Nutritional Composition of Black Soldier Fly (Hermetia Illucens) Prepupae Reared on Different Organic Waste Substrates." Journal of the Science of Food and Agriculture 97, no. 8 (June 2017): 2594-2600. https://doi.org/10.1002/jsfa.8081.

21. Huis, Arnold van. Edible Insects: Future Prospects for Food and Feed Security. FAO Forestry Paper 171. Rome: Food and Agriculture Organization of the United Nations, 2013.

22. Leong, Siew Yoong, Shamsul Rahman Mohamed Kutty, Amirhossein Malakahmad, and Chew Khun Tan. "Feasibility Study of Biodiesel Production Using Lipids of Hermetia Illucens Larva Fed with Organic Waste." Waste Management (New York, N.Y.) 47, no. Pt A (January 2016): 84-90.

https://doi.org/10.1016/j.wasman.2015.03.030.

23. Surendra, K. C., Jeffery K. Tomberlin, Arnold van Huis, Jonathan A. Cammack, Lars-Henrik L. Heckmann, and Samir Kumar Khanal. "Rethinking Organic Wastes Bioconversion: Evaluating the Potential of the Black Soldier Fly (Hermetia Illucens (L.)) (Diptera: Stratiomyidae) (BSF)." Waste Management (New York, N.Y.) 117 (November 2020): 58-80. https://doi.org/10.1016/j.wasman.2020.07.050.

24. Heuel, M., C. Sandrock, F. Leiber, A. Mathys, M. Gold, C. Zurbrügg, I. D. M. Gangnat, M. Kreuzer, and M. Terranova. "Black Soldier Fly Larvae Meal and Fat Can Completely Replace Soybean Cake and Oil in Diets for Laying Hens." Poultry Science 100, no. 4 (April 2021): 101034. https://doi.org/10.1016/j.psj.2021.101034.

25. Schiavone, A., S. Dabbou, M. Petracci, M. Zampiga, F. Sirri, I. Biasato, F. Gai, and L. Gasco. "Black Soldier Fly Defatted Meal as a Dietary Protein Source for Broiler Chickens: Effects on Carcass Traits, Breast Meat Quality and Safety." Animal: An International Journal of Animal Bioscience 13 , no. 10 (October 2019): 2397-2405. https://doi.org/10.1017/S1751731119000685.

26. Neumann, Carmen, Susanne Velten, and Frank Liebert. "N Balance Studies Emphasize the Superior Protein Quality of Pig Diets at High Inclusion Level of Algae Meal (Spirulina Platensis) or Insect Meal (Hermetia Illucens) When Adequate Amino Acid Supplementation Is Ensured." Animals: An Open Access Journal from MDPI8, no. 10 (October 3, 2018). https://doi.org/10.3390/ani8100172.

27. Biasato, llaria, Manuela Renna, Francesco Gai, Sihem Dabbou, Marco Meneguz, Giovanni Perona, Silvia Martinez, et al. "Partially Defatted Black Soldier Fly Larva Meal Inclusion in Piglet Diets: Effects on the Growth Performance, Nutrient Digestibility, Blood Profile, Gut Morphology and Histological Features." Journal of Animal Science and Biotechnology 10 (2019): 12. https://doi.org/10.1186/s40104-019-0325-x.

28. Khamis, Fathiya M., Fidelis L. O. Ombura, Komivi S. Akutse, Sevgan Subramanian, Samira A. Mohamed, Komi K. M. Fiaboe, Weerachai Saijuntha, et al. "Insights in the Global Genetics and Gut Microbiome of Black Soldier Fly, Hermetia Illucens: Implications for Animal Feed Safety Control." Frontiers in Microbiology 11 (2020). https://doi.org/10.3389/fmicb.2020.01538.

29. Erickson, Marilyn C., Mahbub Islam, Craig Sheppard, Jean Liao, and Michael P. Doyle. "Reduction of Escherichia Coli 0157:H7 and Salmonella Enterica Serovar Enteritidis in Chicken Manure by Larvae of the Black Soldier Fly." Journal of Food Protection 67, no. 4 (April 2004): 685-90. https://doi.org/10.4315/0362-028x-67.4.685.

30. Liu, Qiaolin, Jeffery K. Tomberlin, Jeff A. Brady, Michelle R. Sanford, and Ziniu Yu. "Black Soldier Fly (Diptera: Stratiomyidae) Larvae Reduce Escherichia Coli in Dairy Manure." Environmental Entomology 37, no. 6 (December 2008): 1525-30. https://doi.org/10.1603/0046-225x-37.6.1525.

31. Park Soyeon, Hansu Choi, Ji-young Choi, and Gilsang Jeong. "Population Structure of the Exotic Black Soldier Fly, Hermetia Illucens (Diptera: Stratiomyidae) in Korea," December 12, 2017. https://doi.org/10.13047/KJEE.2017.31.6.520.

32. Ståhls, Gunilla, Rudolf Meier, Christoph Sandrock, Martin Hauser, Ljiljana Šašić Zorić, Elina Laiho, Andrea Aracil, et al. "The Puzzling Mitochondrial Phylogeography of the Black Soldier Fly (Hermetia Illucens), the Commercially Most Important Insect Protein Species." BMC Evolutionary Biology 20, no. 1 (May 24, 2020): 60. https://doi.org/10.1186/s12862-020-01627-2.

33. Kaya, Cengiz, Tomas N. Generalovic, Gunilla Ståhls, Martin Hauser, Ana C. Samayoa, Carlos G. Nunes-Silva, Heather Roxburgh, et al. "Global Population Genetic Structure and Demographic Trajectories of the Black Soldier Fly, Hermetia Illucens." BMC Biology 19, no. 1 (May 5, 2021 ): 94. https://doi.org/10.1186/s12915-021-01029-w.

34. Qi, Yingju, Jingyang Xu, Xiaoxuan Tian, Yichuan Bai, and Xishu Gu. "The Complete Mitochondrial Genome of Hermetia Illucens (Diptera: Stratiomyidae)." Mitochondrial DNA Part B 2, no. 1 (January 1, 2017): 189-90. https://doi.org/10.1080/23802359.2017.1307708.

35. Generalovic, Tomas N., Shane A. McCarthy, Ian A. Warren, Jonathan M. D. Wood, James Torrance, Ying Sims, Michael Quail, et al. "A High-Quality, Chromosome-Level Genome Assembly of the Black Soldier Fly (Hermetia Illucens L.)." BioRxiv, November 15, 2020, 2020.11.13.381889. https://doi.org/10.1101/2020.11.13.381889.

36. Zhan, Shuai, Gangqi Fang, Minmin Cai, Zongqing Kou, Jun Xu, Yanghui Cao, Liang Bai, et al. "Genomic Landscape and Genetic Manipulation of the Black Soldier Fly Hermetia Illucens, a Natural Waste Recycler." Cell Research 30, no. 1 (January 2020): 50-60. https://doi.org/10.1038/s41422-019-0252-6.

37. https://reseaucompost.org/

38. https://tinyurl.com/46a7xejr

39. Desjardins, Philippe, and Deborah Conklin. "NanoDrop Microvolume Quantitation of Nucleic Acids." Journal of Visualized Experiments: JoVE, no. 45 (November 22, 2010): 2565. https://doi.org/10.3791/2565.

40. https://tinyurl.com/evaah44c

41. https://www.geneious.com 
42. Katoh, Kazutaka, and Daron M. Standley. "MAFFT Multiple Sequence Alignment Software Version 7: Improvements in Performance and Usability." Molecular Biology and Evolution 30, no. 4 (April 2013): 772-80. https://doi.org/10.1093/molbev/mst010.

43. Maddison, D. R., D. L. Swofford, and W. P. Maddison. "NEXUS: An Extensible File Format for Systematic Information." Systematic Biology 46, no. 4 (December 1997): 590-621. https://doi.org/10.1093/sysbio/46.4.590.

44. Leigh, Jessica W., and David Bryant. "Popart: Full-Feature Software for Haplotype Network Construction." Methods in Ecology and Evolution 6, no. 9 (2015): 1110-16. https://doi.org/10.1111/2041-210X.12410.

45. Cock, Peter J. A., Christopher J. Fields, Naohisa Goto, Michael L. Heuer, and Peter M. Rice. "The Sanger FASTQ File Format for Sequences with Quality Scores, and the Solexa/Illumina FASTQ Variants." Nucleic Acids Research 38, no. 6 (April 2010): 1767-71. https://doi.org/10.1093/nar/gkp1137.

46. Hansen, Kasper D., Steven E. Brenner, and Sandrine Dudoit. "Biases in Illumina Transcriptome Sequencing Caused by Random Hexamer Priming." Nucleic Acids Research 38, no. 12 (July 2010): e131. https://doi.org/10.1093/nar/gkq224.

47. Erlich, Yaniv, Partha P. Mitra, Melissa delaBastide, W. Richard McCombie, and Gregory J. Hannon. "Alta-Cyclic: A Self-Optimizing Base Caller for nextGeneration Sequencing." Nature Methods 5, no. 8 (August 2008): 679-82. https://doi.org/10.1038/nmeth.1230.

48. Jiang, Lichun, Felix Schlesinger, Carrie A. Davis, Yu Zhang, Renhua Li, Marc Salit, Thomas R. Gingeras, and Brian Oliver. "Synthetic Spike-in Standards for RNA-Seq Experiments." Genome Research 21, no. 9 (September 2011): 1543-51. https://doi.org/10.1101/gr.121095.111.

49. Yan, Liying, Mingyu Yang, Hongshan Guo, Lu Yang, Jun Wu, Rong Li, Ping Liu, et al. "Single-Cell RNA-Seq Profiling of Human Preimplantation Embryos and Embryonic Stem Cells." Nature Structural \& Molecular Biology 20, no. 9 (September 2013): 1131-39. https://doi.org/10.1038/nsmb.2660.

50. Meng, Guanliang, Yiyuan Li, Chentao Yang, and Shanlin Liu. "MitoZ: A Toolkit for Animal Mitochondrial Genome Assembly, Annotation and Visualization." Nucleic Acids Research 47, no. 11 (June 20, 2019): e63. https://doi.org/10.1093/nar/gkz173.

51. Dinghua Li, Chi-Man Liu, Ruibang Luo, Kunihiko Sadakane, and Tak-Wah Lam. "MEGAHIT: An Ultra-Fast Single-Node Solution for Large and Complex Metagenomics Assembly via Succinct de Bruijn Graph.” Bioinformatics (Oxford, England) 31, no. 10 (May 15, 2015): $1674-76$. https://doi.org/10.1093/bioinformatics/btv033.

52. Altschul, Stephen F., Warren Gish, Webb Miller, Eugene W. Myers, and David J. Lipman. "Basic Local Alignment Search Tool.” Journal of Molecular Biology 215, no. 3 (October 5, 1990): 403-10. https://doi.org/10.1016/S0022-2836(05)80360-2.

53. Bernt, Matthias, Alexander Donath, Frank Jühling, Fabian Externbrink, Catherine Florentz, Guido Fritzsch, Joern Pütz, Martin Middendorf, and Peter F. Stadler. "MITOS: Improved de Novo Metazoan Mitochondrial Genome Annotation." Molecular Phylogenetics and Evolution 69, no. 2 (November 2013): 313-19. https://doi.org/10.1016/j.ympev.2012.08.023.

54. Leinonen, Rasko, Hideaki Sugawara, Martin Shumway, and International Nucleotide Sequence Database Collaboration. "The Sequence Read Archive." Nucleic Acids Research 39, no. Database issue (January 2011): D19-21. https://doi.org/10.1093/nar/gkq1019.

55. Sudhir, Kumar, Glen Stecher, Michael Li, Christina Knyaz, and Koichiro Tamura. "MEGA X: Molecular Evolutionary Genetics Analysis across Computing Platforms." Molecular Biology and Evolution 35, no. 6 (June 1, 2018): 1547-49. https://doi.org/10.1093/molbev/msy096.

56. Letunic, Ivica, and Peer Bork. "Interactive Tree Of Life (ITOL) v5: An Online Tool for Phylogenetic Tree Display and Annotation." Nucleic Acids Research 49, no. W1 (July 2, 2021): W293-96. https://doi.org/10.1093/nar/gkab301.

57. Suchard, Marc A, Philippe Lemey, Guy Baele, Daniel L Ayres, Alexei J Drummond, and Andrew Rambaut. "Bayesian Phylogenetic and Phylodynamic Data Integration Using BEAST 1.10." Virus Evolution 4, no. 1 (January 1, 2018). https://doi.org/10.1093/ve/vey016.

58. Abrami, Giuseppe, Alexander Mehler, Andy Lücking, Elias Rieb, and Philipp Helfrich. "TextAnnotator: A Flexible Framework for Semantic Annotations," 2019.

59. Hedges, S. Blair, Julie Marin, Michael Suleski, Madeline Paymer, and Sudhir Kumar. "Tree of Life Reveals Clock-like Speciation and Diversification." Molecular Biology and Evolution 32, no. 4 (April 2015): 835-45. https://doi.org/10.1093/molbev/msv037.

60. Tomberlin, J.k., and A. van Huis. "Black Soldier Fly from Pest to 'Crown Jewel' of the Insects as Feed Industry: An Historical Perspective." Journal of Insects as Food and Feed 6, no. 1 (February 6, 2020): 1-4. https://doi.org/10.3920/JIFF2020.0003.

61. Zhang, Shou-ke, Jin-ping Shu, Yang-dong Wang, Ya-ning Liu, Han Peng, Wei Zhang, and Hao-jie Wang. "The Complete Mitochondrial Genomes of Two Sibling Species of Camellia Weevils (Coleoptera: Curculionidae) and Patterns of Curculionini Speciation.” Scientific Reports 9, no. 1 (March 4, 2019 ): 3412. https://doi.org/10.1038/s41598-019-39895-8.

62. Li, Xiao-Dong, Guo-Fang Jiang, Li-Yun Yan, Ran Li, Yuan Mu, and Wei-An Deng. "Positive Selection Drove the Adaptation of Mitochondrial Genes to the Demands of Flight and High-Altitude Environments in Grasshoppers." Frontiers in Genetics 9 (2018): 605. https://doi.org/10.3389/fgene.2018.00605.

63. Peng, Quekun, Lei Tang, Shuai Tan, Zhigang Li, Jifei Wang, and Fangdong Zou. "Mitogenomic Analysis of the Genus Pseudois: Evidence of Adaptive Evolution of Morphological Variation in the ATP Synthase Genes." Mitochondrion 12, no. 5 (September 2012): 500-505.

https://doi.org/10.1016/j.mito.2012.07.107.

64. Gu, Peng, Wei Liu, Yong-fang Yao, Qing-yong Ni, Ming-wang Zhang, Di-yan Li, and Huai-liang Xu. "Evidence of Adaptive Evolution of Alpine Pheasants to High-Altitude Environment from Mitogenomic Perspective." Mitochondrial DNA Part A 27, no. 1 (January 2, 2016): 455-62.

https://doi.org/10.3109/19401736.2014.900667.

65. Zhang, Qi-Lin, Li Zhang, Tian-Xuan Zhao, Juan Wang, Qian-Hua Zhu, Jun-Yuan Chen, and Ming-Long Yuan. "Gene Sequence Variations and Expression Patterns of Mitochondrial Genes Are Associated with the Adaptive Evolution of Two Gynaephora Species (Lepidoptera: Lymantriinae) Living in Different High-Elevation Environments." Gene 610 (April 30, 2017): 148-55. https://doi.org/10.1016/j.gene.2017.02.014.

66. Luo, Yongjun, Xiaohong Yang, and Yuqi Gao. "Mitochondrial DNA Response to High Altitude: A New Perspective on High-Altitude Adaptation." Mitochondrial DNA 24, no. 4 (August 1, 2013): 313-19. https://doi.org/10.3109/19401736.2012.760558.

Page $10 / 17$ 
67. Russo, Claudia A. M., Beatriz Mello, Annelise Frazão, and Carolina M. Voloch. "Phylogenetic Analysis and a Time Tree for a Large Drosophilid Data Set (Diptera: Drosophilidae)." Zoological Journal of the Linnean Society 169, no. 4 (2013): 765-75. https://doi.org/10.1111/zoj.12062.

68. Zhou, Fen, Jeffery K. Tomberlin, Longyu Zheng, Ziniu Yu, and Jibin Zhang. "Developmental and Waste Reduction Plasticity of Three Black Soldier Fly Strains (Diptera: Stratiomyidae) Raised on Different Livestock Manures.” Journal of Medical Entomology 50, no. 6 (November 2013): 1224-30. https://doi.org/10.1603/me13021.

\section{Tables}

Table 1 : Origin of $\mathrm{CO1}$ sequences and mitochondrial genomes

\begin{tabular}{lll} 
Origin & Number of individuals (CO1) & Number of individuals (Mitochondria) \\
\hline Collection from the wild & 91 & 43 \\
\hline Industrial origin & 23 & 13 \\
\hline Mined from databases (GenBank \& Bold) & 560 & 1 \\
\hline Assembled from SRA (raw sequences) & 3 & 3 \\
\hline Total & 677 & 60
\end{tabular}

Table 2: Geographic origin of $\mathrm{CO} 1$ sequences and mitochondrial genomes

\begin{tabular}{lll} 
Geographic origin & Number of Individuals - CO1 & Number of Individuals - mitochondria \\
\hline South America & 55 & 4 \\
\hline North America & 43 & 2 \\
\hline Asia & 277 & 4 \\
\hline Oceania & 106 & 0 \\
\hline Africa & 78 & 11 \\
\hline Europe (including France) & $118(90)$ & $39(38)$ \\
\hline Total & 677 & 60
\end{tabular}

\section{Figures}




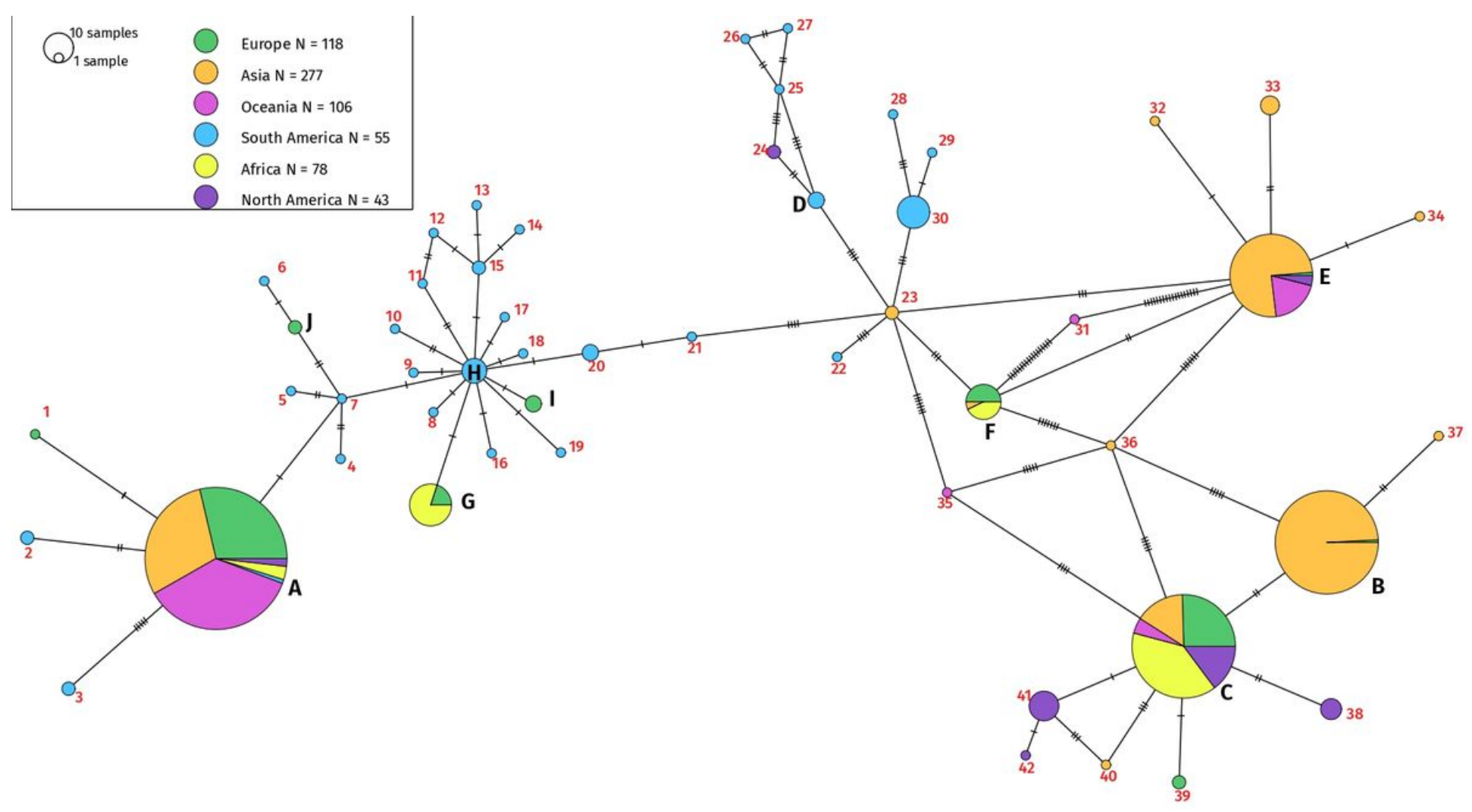

Figure 1

C01 haplotype network of Hermetia illucens

The haplotype network generated by PopArt after multiple alignments are based on the $\mathrm{CO} 1$ gene of Hermetia illucens. Analysis of the differences between the C01 gene sequences of 677 individuals. The pie charts represent the same haplotype, and their relative size indicates the number of individuals sharing this haplotype. The number of bars between each segment indicates the differences between the two sequences. Within a pie chart, the colors represent the geographical origin of the individuals. The major haplotypes are more than five sequences (A B C E F G H 3038 \& 41). The minor haplotypes are the others. The list of sequences and their exact origin is available as an additional document. 


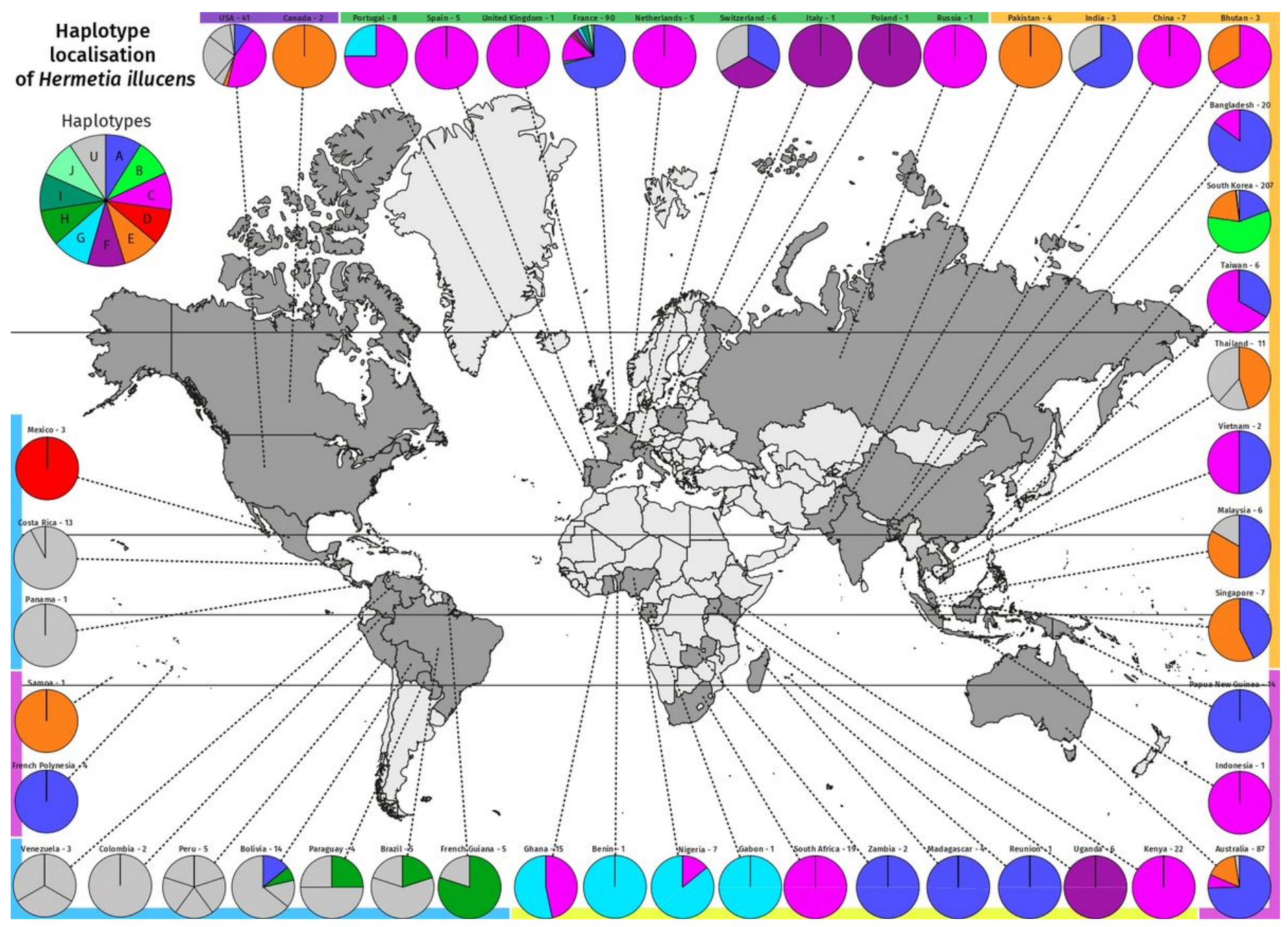

Figure 2

World C01 haplotype distribution

Global distribution of the different Hermetia illucens haplotypes based on the $\mathrm{CO1}$ gene. The pie charts represent the proportion of each haplotype within a country. The country's name is followed by the number of individuals analyzed. A colored band representing the continents is next to each pie chart. The colors represent the haplotypes determined in figure 1 . The grey haplotypes are represented with a number in figure 1 . Separation is made in the pie chart when the haplotypes change for the same country. For example, Venezuela contains three unique haplotypes, meaning that these three individuals are different. A summary table can be found in supplementary table 3 . 


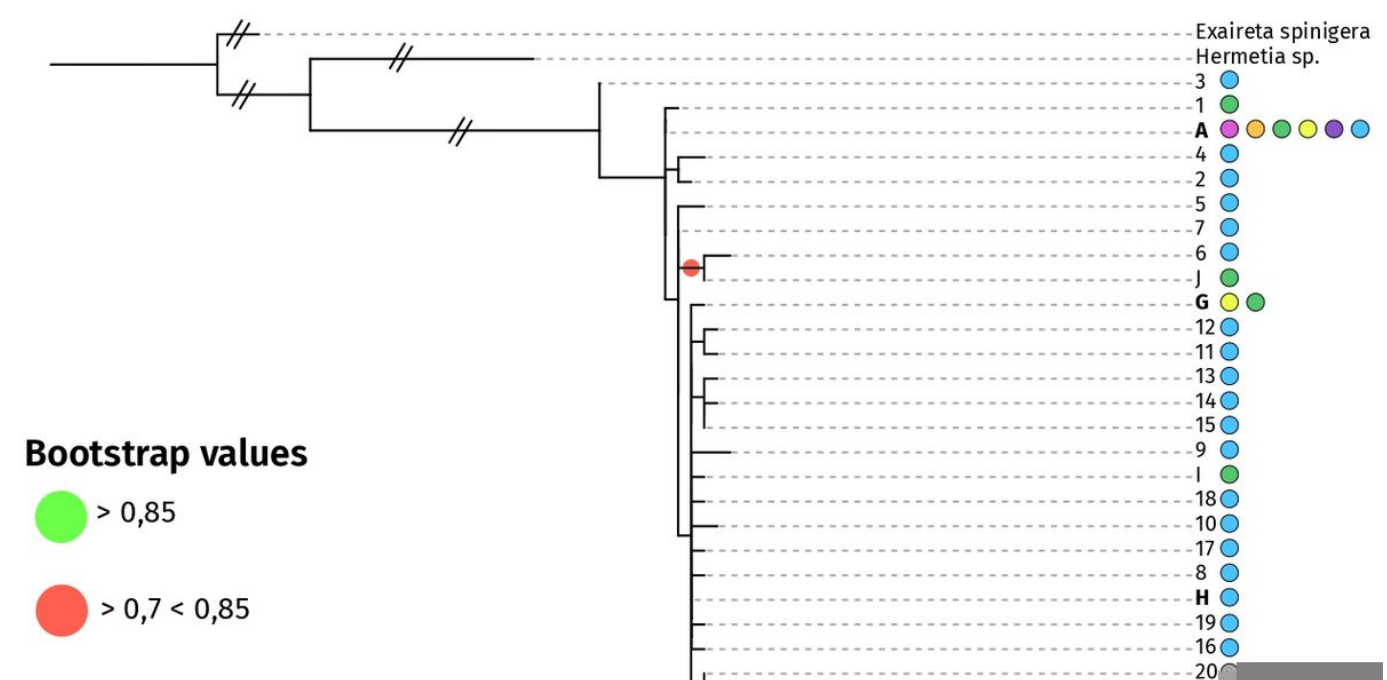

\section{Figure 3}

c01-based phylogenetic tree

ML phylogenetic tree. Rooted phylogeny with Exaireta spinigera (Stratiomyidae). Bootstrap values are materialized with colored circles: green for values $>0.85$ and red for values between 0.7 and 0.85 . We only kept one sequence per haplotype to visualize the relationship between them. Next to each haplotype, colored circles represent the geographical origin of the species present in this haplotype. The order indicates the relative abundance of the individuals of a container in each haplotype. The statistical support was estimated with 500 Bootstraps with MegaX software using the Tamura $92 \mathrm{G}+\mathrm{I}$ model. 


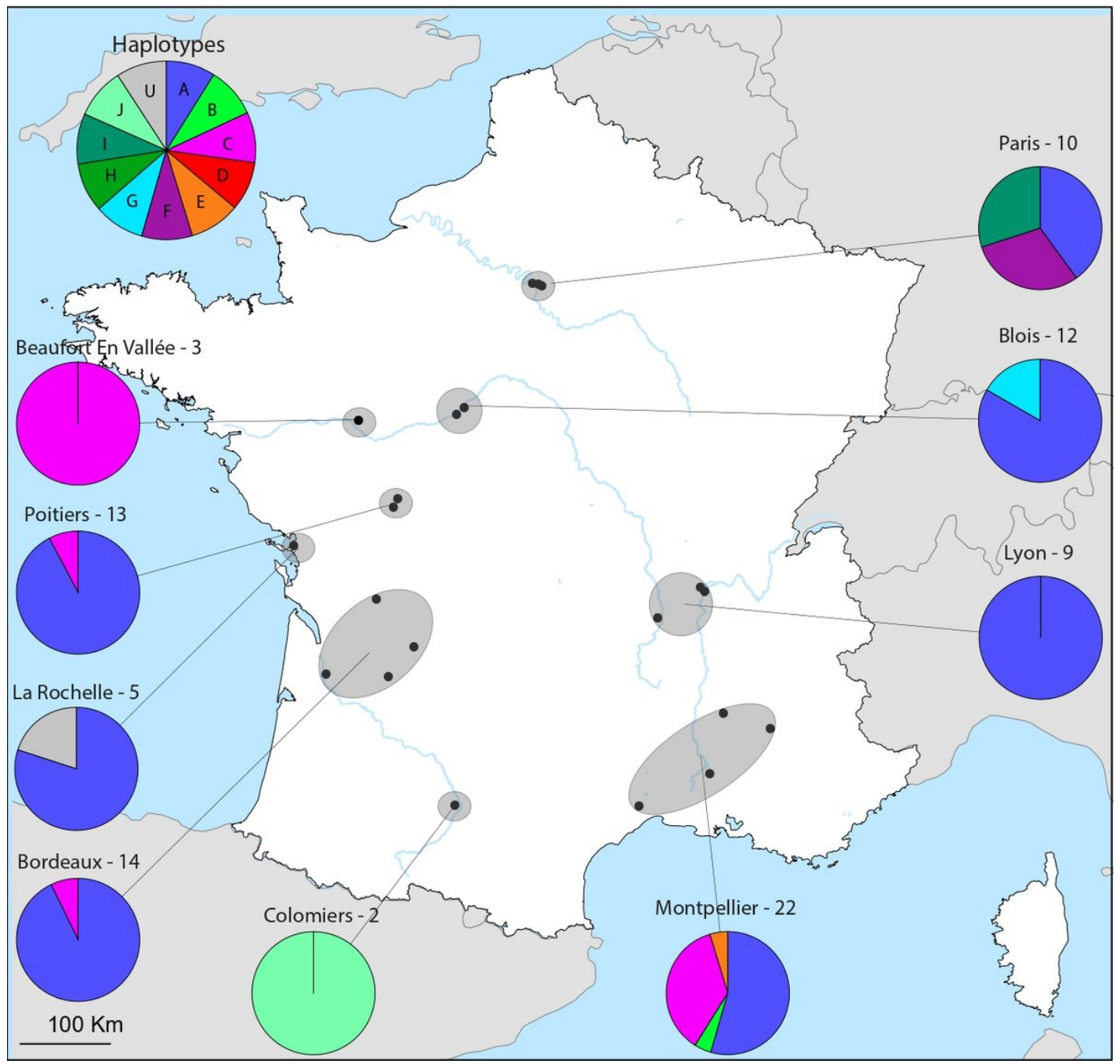

\section{Figure 4}

France C01 haplotype distribution

Distribution of the different haplotypes of Hermetia illucens in France. The dots represent the specific area of collection. The pie charts represent the distribution of haplotypes in the corresponding area. The names of the main cities in each area are indicated above and the number of sequenced individuals.

Paris includes Vincennes and Nogent Sur Marnes. Blois includes Valloire Sur Cisse. Lyon includes Saint Marcellin En Forez, Corbas, Champagne Au Mont d'Or and Dardilly. Montpellier includes Avignon, Trescléoux and Dieulefit. Bordeaux includes Lormont La Force and Périgueux. Poitiers includes Saint Georges Lès Baillargeaux. 


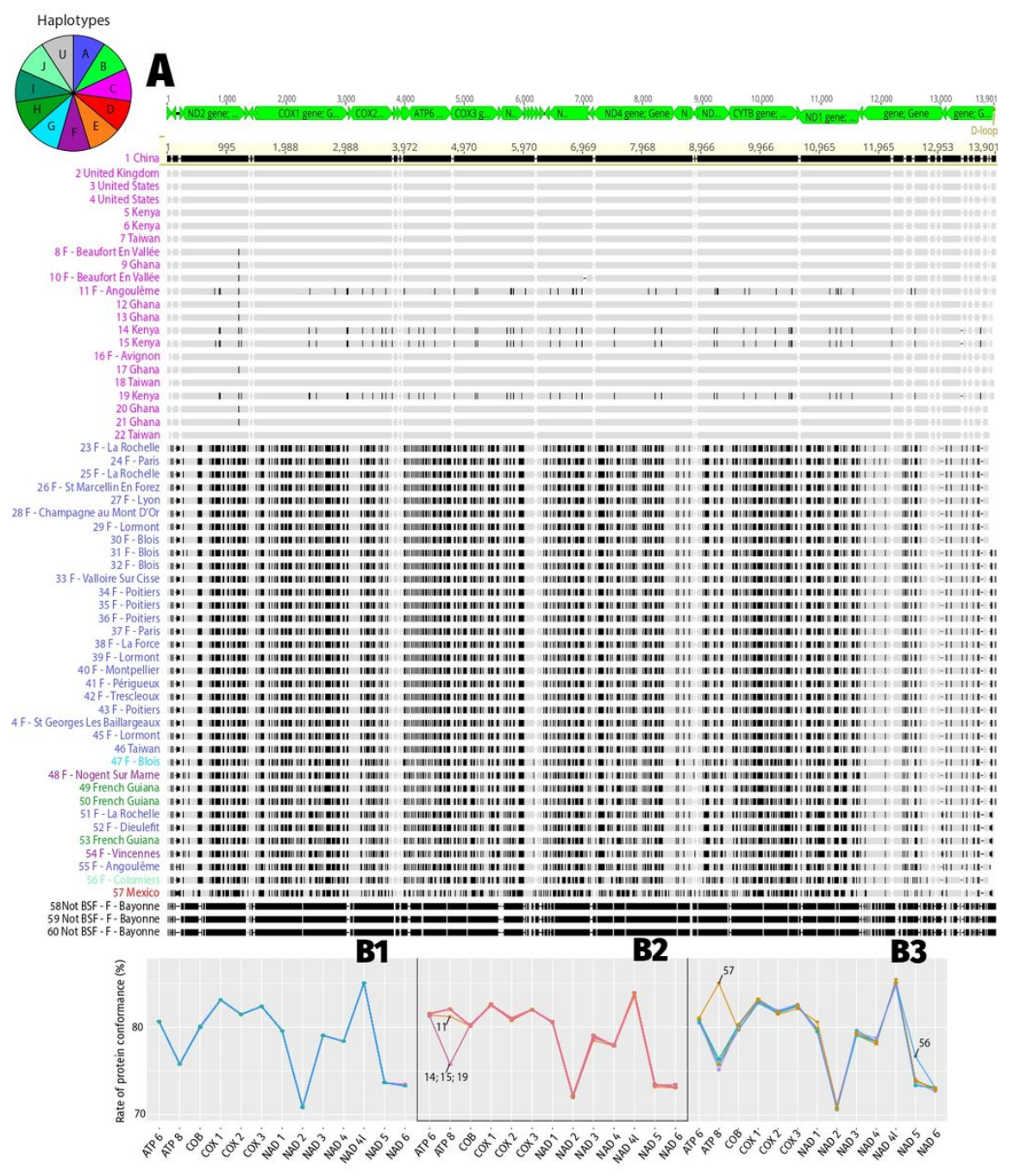

\section{Figure 5}

\section{A: Mitochondrial DNA alignment}

Multiple alignments of mitochondrial DNA sequences from Hermetia illucens. Alignment was performed with the MAFFT aligner. The colors of the names come from their haplotypes determined with the $\mathrm{CO} 1$ sequences. Each black mark represents a difference with the reference (1 - China). Above the alignment, there is a representation of the mitochondrial genes.

\section{B: Sequence conformance between Hermetia illucens and Exaireta spinigera}

Sequence conformity of mitochondrial genes of Hermetia illucens to Exaireta spinigera. The sequences are grouped according to haplotypes. The A haplotype (B1), the $C$ haplotype (B2), and the other haplotypes (B3). The sequences inside each graph are represented in the alignment (5A). Sequences from 23 to 46 plus sequences 51,52 , and 55 for haplotype A (5-B1); from 1 to 22 for haplotype $C$ (5-B2); and from 47 to 57 (except sequences 51 , 52, and 55) for the other haplotypes (5-B3). 


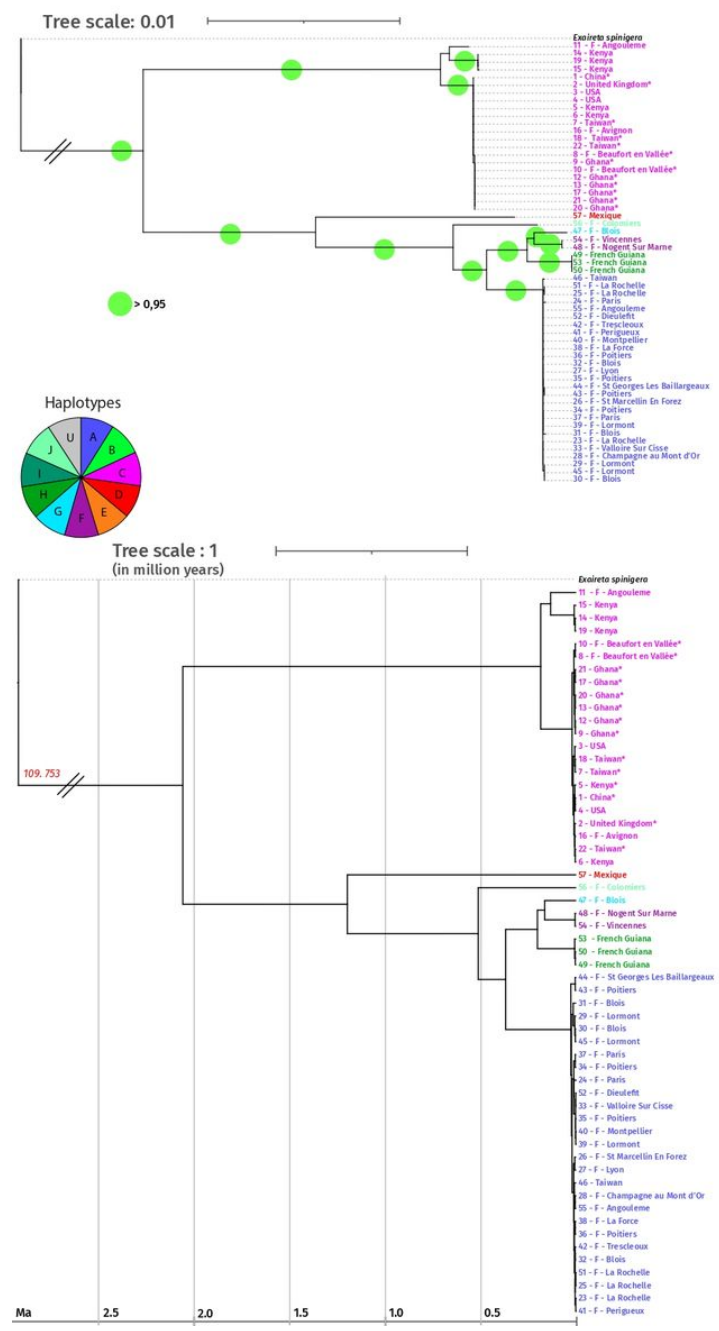

Figure 6

6A: Mitochondrial DNA phylogenetic tree

ML phylogenetic tree, of 57 mitochondrial DNA sequences of Hermetia illucens, deprived of D-Loop regions. MAFFT was used to align the sequences, 500 Bootstrap replicas were performed. The outgroup used is Exaireta spinigera (Stratiomyidae). The colors of the names correspond to the haplotypes determined with the $\mathrm{CO} 1$ gene sequences (Figure 1). Bootstrap values are indicated by a green circle when they are $>0.95$. Commercial individuals are marked with an asterisk.

B Mitochondrial DNA time tree

Bayesian time tree obtained with BEAST of 60 mitochondrial genomes of Hermetia illucens rooted with Exaireta spinigera. The scale is in millions of years. A break in scale is made at the root level. The colors of the names correspond to the haplotypes determined with the CO1 sequences (figure 1). Commercial individuals are marked with an asterisk.

\section{Supplementary Files}

This is a list of supplementary files associated with this preprint. Click to download.

- S001.xlsx

- S002.pdf

- s003.pdf

- s004.pdf

- S005.xlsx

- S006.pdf 